\title{
Presencia en la Ausencia: El Juego como Instrumento en un Taller Artístico de Enfoque Terapéutico en la Casa Familiar Dr. Juan Segura
}

Olga Leyva Gutiérrez - Universidad de Granada

Elizaberta López Pérez - Universidad de Granada

Recepción: 10.05.2021 | Aceptado: 14.06.2021

Correspondencia a través de ORCID: Olga Leyva Gutiérrez
0000-0003-1870-1588

0000-0003-0283-6752

Citar: Leyva Gutiérrez, O y López Pérez, E (2021). Presencia en la Ausencia: El Juego como Instrumento en un Taller Artístico de Enfoque Terapéutico en la Casa Familiar Dr. Juan Segura. REIDOCREA, 10(23), $1-14$.

Estudio de investigación de Trabajo Final de Grado

Área o categoría del conocimiento: Arteterapia

Resumen: Se presenta la experiencia de un taller de arteterapia realizado en la Casa Familiar Dr.Juan Segura. Esta investigación gira en torno a lo ocurrido con uno de los participantes (50 años), y el modo de establecer con él un vínculo terapéutico que favorezca su creatividad y a través de ella, la conciencia sobre su propia historia. Se infiere un proceso de arteterapia a partir del juego y el acompañamiento, usando específicamente la técnica del Garabato de Winnicott, lo que permite reforzar los vínculos hacia la actividad y personas implicadas en el proceso, reconociendo los fenómenos transicionales presentes durante la experiencia. Los resultados afirman la hipótesis de partida, ya que gracias al arteterapia se logra la activación de la creatividad del usuario, dando lugar al descubrimiento de un nuevo alfabeto de formas en la producción de autoría compartida y sobre todo, a la posibilidad de reconocerse como sujeto de su propia vida.

Palabra clave: Arteterapia

Closing the patient's affective gap: Play as an Instrument to promote Creativity and Self-Awareness in a Therapy-Oriented Art Workshop at the Dr Juan Segura Care Centre

Abstract: This study reports on the findings of an art therapy workshop conducted at the Dr Juan Segura Care Centre - a mental health care facility providing day and permanent housing services to the mentally handicapped in Granada City (Spain). Specifically, the research documents our experience in interacting with one male workshop participant (aged 50), who was approached by the therapist with a view to tightening emotional bonds with him. The ultimate goal in doing so was to stimulate the patient's creativity, and eventually, make him aware of his life experience. The art therapy method that was applied is Winnicott's Squiggle Game. This technique reinforces the patient's engagement with the drawing activity, and requires the therapist's full involvement, which enriches the patient-therapist rapport. For this reason, the Squiggle Game enables the identification of the transitional processes and phenomena occurring throughout the exchange. The results of this study proved the initial hypothesis right. Accordingly, art therapy is shown to be highly instrumental in triggering the creativity of patients, who acquire a wider range of drawing patterns and strategies during the therapeutic interplay. Most importantly, art therapy is found to help patients build their self-awareness and consciously experience their individuality.

Keyword: Artherapy

\section{Presentación}

Esta investigación surge a partir de la experiencia de un taller de arteterapia desarrollado en la Casa Familiar Dr. Juan Segura (Granada, España), durante los meses de febrero a mayo de 2019, como desarrollo de la asignatura Prácticas Externas del Grado en Bellas Artes. Es una de las sedes de la Fundación Cruz Blanca dedicada a la atención integral para personas con discapacidad intelectual y problemas de conducta. Su misión es prestar un servicio individualizado, a través de un repertorio de actividades y experiencias que fomenten la autonomía e integración de esas personas y sus familias. Cuentan con una amplia variedad de actividades a elegir, dependiendo del estado y necesidad de cada usuario/a. Entre estas se encuentra el taller de arteterapia, que, según la dirección del centro, ayuda notablemente a gran parte de los asistentes. No es 
la primera vez que se acoge este taller para incorporarlo a sus actividades; su trayectoria y sus buenos resultados desde 2016 han hecho que se llegue a convertir en una de las sedes habituales para las Prácticas Externas de la Facultad de Bellas Artes de Granada.

Durante esta experiencia, se desempeñará el papel de guía del taller de arteterapia, con la supervisión estrecha desde el entorno universitario de la profesora y arte-terapeuta titulada, Elizaberta López Pérez, además de la colaboración del equipo clínico y asistencial del centro. A lo largo del proceso se trabajará con los/as participantes del taller constatando cómo algunos de los fundamentos del arteterapia se hacen patentes.

Aunque analizaremos cómo se ponen en juego dichos parámetros más detenidamente, en primer lugar queremos destacar el valor inestimable del apoyo de la entidad de prácticas. En efecto, el centro Casa Familiar Dr. Juan Segura está comprometido a facilitar el desempeño del taller, ayudando a que se respete, por ejemplo, el imprescindible encuadre terapéutico. El término encuadre, se refiere a las "reglas explícitas que regulan el encuentro clínico y a las premisas que derivan de la comprensión teórico-técnica que se aplican a dicho encuentro, éste hace posible la construcción del vínculo terapéutico (implicación mutua de ambos partícipes para el cambio)"(Ávila Espada, 2015, p.395). En nuestro caso, este encuentro, entendido como un acompañamiento en el proceso creador, partirá de un acuerdo entre el/la guía del taller y el asistente, que deberá ser respetado tanto por sus participantes como por el entorno que rodea al taller. Por ello es fundamental la implicación de la institución para el desarrollo de la experiencia.

En la Casa Familiar viven treinta personas, de las que el equipo clínico seleccionó diez. Son usuarios/as muy institucionalizados que tienen pocas salidas al exterior, excepto las que son justificadas por razones de asistencia a actividades externas. Las visitas de sus familiares no son demasiado abundantes. Es por esta razón que este tipo de actividades, como el taller, que supone la incorporación y renovación semestral de personas procedentes de otros ámbitos, como es el caso de las estudiantes de Bellas Artes, genera mucha expectación entre los/as residentes.

Con esta investigación se muestra parte del trabajo realizado por una de estas estudiantes, centrándonos en un caso sobresaliente (Pe a partir de ahora).

Las ideas fundamentales que se van a analizar se focalizan en:

- El valor del proceso creador.

- El carácter dinámico de la investigación, centrado en el acompañamiento y la observación.

- El juego como metodología de trabajo (Garabato de Winnicott).

Pero si hay un concepto que reúna a los otros este es, sin duda, la cualidad procesual de la experiencia arteterapéutica: la creación entendida desde el proceso, más que en la búsqueda de un resultado final, (propia de actividades artísticas enfocadas al mercado) enaltece su carácter dinámico, y hace posible el encuentro intersubjetivo necesario en arteterapia, y por tanto, el acompañamiento, la observación y el juego.

Cercano a la vida, el proceso arteterapéutico nos permite adoptar las palabras de Antonio Machado (1912): "Caminante, son tus huellas el camino y nada más; caminante no hay camino, se hace camino al andar." Esta investigación nos confirmará, que son las huellas que nos dejamos los seres humanos, unos a otros, las que nos construyen y dibujan el camino por donde transitar. Pe dejó las suyas en nosotras, estas que exponemos aquí, y somos lo que somos ahora, también gracias a ellas.

\section{Objetivos}


Los objetivos se plantean partiendo de la hipótesis:

El arteterapia es un vehículo posible para el establecimiento de un vínculo simbólico, en especial con una dinámica de juego según la técnica del garabato de Winnicott, en un contexto de discapacidad intelectual y problemas de conducta.

Por esto, nos planteamos los siguientes objetivos:

- Inferir un proceso de arteterapia a partir del juego del garabato de Winnicott.

- Acompañar en el proceso arteterapéutico para que el usuario se sienta sostenido en su posible vivencia de carencia y/o escasez de apego.

- Establecer vínculos transferenciales entre usuario y guía del taller de arteterapia mediante el reconocimiento de fenómenos transicionales.

- Utilizar el juego como estrategia para reforzar los vínculos hacia la actividad y las personas implicadas en el proceso.

\section{Características generales del colectivo}

Los/as asistentes del taller representan en torno al $20 \%$ de los/as residentes en la Casa Familiar Dr Juan Segura. Estos/as son elegidos/as por los/as profesionales del centro en función de sus necesidades y características de conducta, priorizando a aquellas personas que tienen especial disposición hacia la actividad artística, las que tienen dificultades de comunicación verbal y/o de relación social. En definitiva, todas aquellas que pudieran beneficiarse de ello, pero, como ellos/as mismos/as manifiestan, teniendo cuidado de evitar en lo posible situaciones disruptivas. La realidad del taller nos enseña que esta última condición no es del todo previsible, pues tenemos evidencias de personas que en este contexto abandonan este tipo de actitudes mostrándose perfectamente integrados/as.

Aunque la selección de asistentes se limite a unos diez, el resto de quienes habitan en la Casa Familiar, manifiestan en ocasiones su deseo de disfrutar del hacer artístico. Es por esto que dentro del encuadre temporal, se cuenta con una franja de asistencia libre a la que acuden usuarios/as interesados/as. Esta dinámica posibilita observar la aceptación que tiene el taller tanto para las personas seleccionadas como para el resto, pudiendo comprobar el éxito de la actividad entre los/as asistentes.

La intención del taller de arteterapia será la de dar una atención individualizada a todos sus usuarios/as, para que puedan disfrutar de los beneficios del hacer artístico desde la perspectiva terapéutica, que según la Asociación de Arteterapia debe apuntar a enriquecer su calidad de vida (AATA, 2003 como se citó en López y Martínez, 2006, p.41).

Pero ¿en qué se materializa esto? O lo que es lo mismo, ¿de qué forma podemos desde el taller aspirar a enriquecer su calidad de vida? Sabemos que este colectivo está muy bien atendido desde un punto de vista social, emocional y físico en la Casa Familiar. Lo que parece ausentarse, o al menos estar poco presente, es la vida que sucede en el exterior, como su dimensión familiar y privada. Es cierto que la entidad vigila de cerca el cuidado emocional, posibilitando las visitas familiares, las interacciones con lo externo, promoviendo la participación de los/as residentes en la medida de lo posible en vivencias que impliquen contacto con el exterior. A pesar de ello, la realidad de su cotidianeidad es que la mayor parte del tiempo la vida se desarrolla en la Casa, que se establece realmente como su domicilio estable y espacio habitual. Es un lugar muy cómodo, muy bien habilitado, pero no puede aspirar a lo que sería la vida privada particular de sus residentes, si no estuvieran condicionados/as por sus peculiaridades 
conductuales e intelectuales, y eso es algo que no escapa a un buen número de ellos/as, que manifiestan su añoranza hacia la casa familiar, sea esta real o imaginada.

El taller les ofrece la posibilidad de dar cierta realidad a estos aspectos a través de las obras, además de ponerlos en diálogo con el grupo y con la guía de arteterapia, dándoles presencia. Su propia historia se pone en valor y se pueden convertir en protagonistas de ella. Esto da realidad a los hechos vividos, los recuerdos y también los deseos y temores, haciendo posible pensarse dentro de su relato. Es una forma de situarse como sujetos creadores de sí mismos/as, contribuyendo a su propio devenir y, de alguna manera, un lugar de resistencia a la marginación que no pueden esquivar del todo, a pesar de estar minimizada por la dedicación y el buen hacer de esta entidad y sus profesionales.

\section{Encuadre}

El encuadre es un requisito indispensable para el taller. Se trata de una matriz grupal definida por Masini Fernández (2015, p.156-157) como una "incubadora psicoterapéutica, que facilita el tránsito hacia un desarrollo mejor". Un espacio entendido por esta autora como aquel que "tiene valor simbólico, sagrado, donde va a representarse el mundo interno de los que en ella participan”. En la práctica, consiste en poner un marco que defina cuatro aspectos fundamentales, tal y como manifiestan Avilés Martos y López Pérez (2014, p.76): "Este encuadre se genera desde la primera sesión, donde se establecerán las reglas básicas del proceso de Arteterapia, fundamentado en establecimiento de unos límites en términos de respeto, referido al espacio, temporalización, los roles y la tarea".

En nuestro caso se va a concretar en:

- Tiempo: El taller se desarrolla los lunes y jueves de 17:00h a 19:00h. Dentro de ese tiempo se establece una estructura:

- De 17:00h a 17:30h: Presentación de la actividad y, si procede, exposición de las obras tal y como habían quedado el último día, y comentarios del autor/a de forma voluntaria.

- De 17:30h a 18:30h: Trabajo artístico.

- De 18:30h a 19:00h: Asistencia libre.

- Espacio: El taller se desarrolla en una sala multidisciplinar dentro de la Casa Familiar, que además dispone un patio exterior que le otorga luminosidad a la estancia, una pila de lavado, caballetes, mesas, sillas, muebles y estanterías, además de un almacén independiente. En esta misma sala, se comparte el espacio con una zona de despacho que frecuentan monitores del centro. Su función aquí es supervisar el comportamiento de los/as asistentes, pero en ocasiones su presencia interfiere en la actividad, ya que cualquier ruido o actividad que se esté llevando a cabo de forma paralela al momento de producción, les distrae y altera notablemente.

- Rol: El papel que ocupa la guía del taller en este caso, no es el de arteterapeuta, sino el de facilitadora de los procesos. Para desempeñar el rol de arteterapeuta se necesita de una titulación en un Máster de Arteterapia ${ }^{1}$. Sin embargo, gracias

\footnotetext{
${ }^{1}$ Algunos de los Máster de Arteterapia acreditados por la Federación Española de Asociaciones de Profesionales de Arteterapia (http://feapa.es/formaciones/) son: Universidad Complutense de Madrid: Máster Oficial de Arteterapia Interuniversitario (conjunto con UAM y UVA) Universitat de Girona: Máster Integrativo en Arteterapia Universidad de Murcia: Máster en Arteterapia y Mediación Plástica.
} 
a su formación en Bellas Artes, es conocedora del proceso artístico, lo que facilitará el acompañamiento en la producción. Por otro lado, no hay que olvidar que la experiencia se desarrolla, como ya se ha indicado, dentro de la asignatura Prácticas Externas del Grado en Bellas Artes, con lo cual cuenta con el asesoramiento, en primer lugar, de la tutora académica, Elizaberta López que además de profesora de la Facultad de Bellas Artes es arteterapeuta titulada, y, en segundo lugar, de los/as profesionales del centro (psicóloga, trabajadora social, terapeutas, monitores/as). Esto evitará las interferencias con las dinámicas específicas del centro para con cada usuario/a y, aún con más determinación, la intrusión en cuestiones complejas que no son competencia de este taller. El papel de acompañante se desempeñará de un modo "no invasivo, sino sutil, discreto y sobrio desde una presencia activa, ajustada e intuitiva" (Bassols, 2006, p.23), con un especial cuidado de que no se confunda con una relación personal diferente a la que se propone. Debe quedar claro en el acuerdo inicial con los/as participantes, cuál es el rol del guía, que no se acerca a aquel que desempeña un/a amigo/a, un/a psicólogo/a, un/a asistente del centro, etc. Una relación aparte de la vida privada y personal.

- Tarea: Lo que lleva tanto a los/as usuarios/as como a la guía a estar en ese espacio y tiempo, es el fin de desarrollar un proceso artístico en el contexto de una relación vincular acompañante y desde un enfoque arteterapéutico. En dicho proceso pueden emerger cuestiones que forman parte de la vida del/de la usuario/a transferidos al hacer artístico; esto es lo que proporciona la contingencia de lo terapéutico. La función no es la que desempeña una profesora de arte, por lo que no se siguen pautas que tengan que ver con lo educativo, ni con el aprendizaje de técnicas artísticas. No se busca un tratamiento ni mucho menos un diagnóstico; la producción no se confunde con la persona. El enfoque en la tarea es fundamental porque mantiene los límites establecidos en el encuadre: la experiencia transcurre en el espacio temporal y físico del taller y con una persona como guía facilitadora del proceso. Fuera de este marco no existe la experiencia, por lo que no excede a otros tiempos ni espacios fuera de este, ni tiene que extenderse tampoco la relación intersubjetiva.

Este pacto entre el/la participante y el/la guía se mantendrá durante toda la experiencia (López y Martínez, 2006), al igual que los/las participantes, para otorgarle así una mayor estabilidad.

\section{Experiencia del taller en el protagonista Pe}

Durante el desarrollo del taller hay un caso sobresaliente que es Pe. Nos informan desde la dirección del centro que tiene en torno a cincuenta años, así como de otros aspectos que debemos conocer para comprender su forma de ser y de expresarse.

Universidad Pablo de Olavide, Sevilla: Máster en Arteterapia y aplicaciones del Arte para el Diálogo y la Integración Social. Universitat Politècnica de València: Máster en Arteterapia. Aspectos educativos, sociales y terapéuticos del Arte como experiencia de creación acompañada. 

6

Es una persona muy activa que habla especialmente deprisa, pero a pesar de esto se le entiende suficientemente bien². Tiene un acento propio del entorno rural al que pertenece, un pueblo de Granada, que caracteriza expresiones que repite una y otra vez de forma muy peculiar y estereotipada. Transmite una cercanía especial en sus relaciones sociales, repitiendo a su interlocutor/a que "le quiere mucho" y enseñando sus objetos más preciados, sin perder la oportunidad de pedir a cambio "un caramelo" o algún objeto por el que muestra afinidad. Observamos como le satisface tener presentes en su diálogo a su figura fraterna (su hermana $\mathrm{C}$, a partir de ahora), y a los objetos que le recuerdan su relación con ella. La Casa Familiar nos informa de esta repetición obsesiva y el deseo de poseer todos los objetos posibles, que cada cierto tiempo deben revisar y requisar por razones de higiene. Parecen convertirse en elementos que palian el dolor que le provoca el distanciamiento con su hermana; es posible que tengan que ver con lo que Abadi (2014) nos confirma: "el aparato psíquico se verá dificultado... y el niño o adulto tendrán una tendencia a buscar objetos concretos de los que dependerá adictamente para acabar con el sentimiento de vacío o soledad" (p. 23). Pero como sigue diciendo la autora, en estos casos puede que ya "no sirvan para elaborar la ausencia, si no solo para negarla" (Abadi, 2014, p.23).

Su discurso es algo limitado, habla sin parar de su hermana $\mathrm{C}$ y de aquello que los une, principalmente una casa que desearía que compartieran. Nos llama la atención esta actitud entre el resto de los usuarios/as, ya que además de tener una forma de ser un tanto peculiar, deducimos que a través de esta repetición parece conseguir mantenerla presente de forma continua. Expresiones como "la echo de menos", "¿Cuándo viene mi hermana C?”, “¿Me has traído caramelos como los que me trae mi hermana?”, nos confirman que la añora permanentemente. Quizás sea el modo de mantener en el presente su historia pasada antes de entrar al centro. Es su forma de llenar la ausencia que se crea en el distanciamiento con la vida exterior.

Entre todas las producciones que se llevan a cabo en el taller, la de Pe es de las más sorprendentes. Esa repetición constante de sus expresiones se manifiesta también a través de la creación artística como estereotipia dibujada. La "casa de mi (su) hermana $C$ " aparece tantas veces que resulta inquietante, siempre con sus ventanas, puerta con escalinata-camino de acceso, patio lateral con flores, y una chimenea de la que emerge una escalera-humo infinita, con su doble especular al otro lado del tejado. Ventanas y puertas aparecen con un aspa inscrita, como si con eso quisiera enfatizar su presencia, o clausurarlas de forma contundente, marcarlas, de manera similar al modo que cierra habitualmente sus expresiones verbales con la palabra " Así es!"

Una y otra vez, la casa se convierte en el motivo de la representación, sin mostrar la más mínima preocupación por ampliar la variedad de formas. "Lo que más me gusta dibujar es la casa de mi C", dejándonos claro lo que significa para él.

Estas estereotipias, tanto verbales como gráficas,

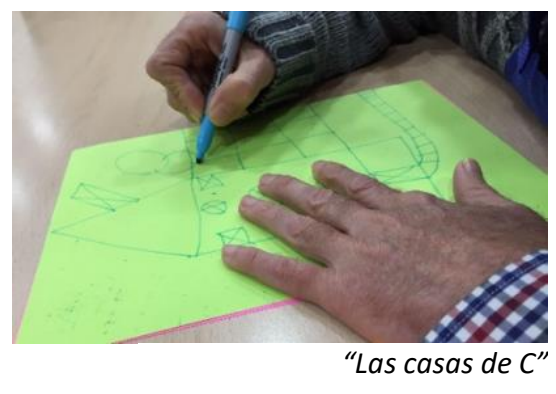
podrían identificarse como "actos reparadores convertidos en repeticiones compulsivas

\footnotetext{
${ }^{2}$ En este contexto no es siempre así, dándose el caso de residentes que no tienen comunicación verbal o la que tienen es ininteligible. Por eso nos parece destacable este detalle.
} 
y obsesivas" (Laplanche y Pontalis, 1996, p.336). Tras revisar varias concepciones acerca de lo que puede significar esta repetición, nos decantamos por teorías como la de Viktor Lowenfeld (1980), entendiendo la repetición como un "significado de carencia afectiva y la incapacidad de asumir sentimientos y emociones concretas" (Ortega, 2014, p.134). Asociamos esto último a $\mathrm{Pe}$, ya que parece mostrarse reacio a asimilar ciertas emociones, pudiéndose observar cómo a través de esta repetición, parece reparar, y con ello, enmascarar su situación emocional (Lowenfeld y Brittain, 1980), aliviando su sentimiento de desamparo.

En una de las sesiones el usuario descubre un objeto que pertenece a la guía del taller, "una pulsera", que llama especialmente su atención y comienza a pedir con insistencia que se la regale. Esta petición se convierte en cuestión de minutos en una obsesión, que acaba siendo lo que parece una necesidad vital. Su estado de ánimo cambia, su nerviosismo va en aumento, y esto produce un efecto visible de forma inmediata en su producción. Se observa como su estado de ánimo marca las principales diferencias

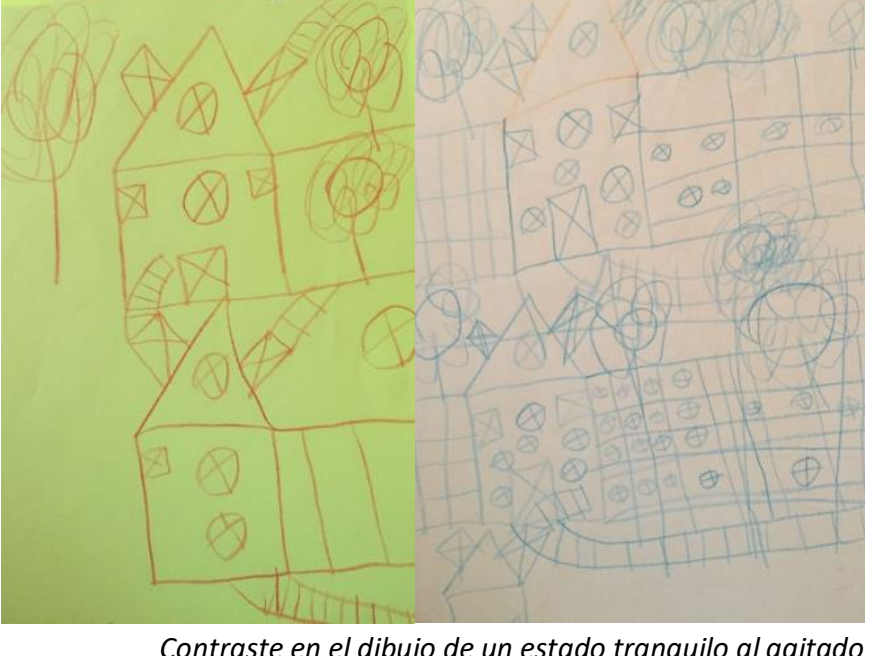

Contraste en el dibujo de un estado tranquilo al agitado formales. La repetición de elementos se acentúa, llegando incluso a solaparse dentro del mismo formato.

Marian López y Noemí Martínez (2006) hablan de la casa como una imagen en la que es común la proyección de la persona que dibuja. En una misma sesión hemos podido observar que, al comenzar a alterarse por el deseo de conseguir algo, ha pasado de su representación ordenada como acostumbra a la necesidad de repetir profusamente algunos elementos, en especial los signos destinados habitualmente a las ventanas. Parece que los hubiera resignificado, al ritmo que paralelamente verbaliza el objeto de su deseo. Nos preguntamos si ahora, además de ventanas, son pulseras. Además, si estas casas, como nos confirman las autoras, remiten a sí mismo, estamos siendo testigos del registro visual de su estado emocional.

Entretanto, sigue insistiendo. Parece dispuesto a hacer cualquier cosa para obtenerla. Su vehemencia empieza a crear un ambiente de cierta inestabilidad entre los/as demás asistentes del taller. En cierto modo obligada por la situación, la guía del taller termina cediendo y dándole el objeto que tanto desea, y su agradecimiento es inmenso. "Gracias, gracias de verdad por darme la pulsera", repite Pe una y otra vez.

Este hecho y el efecto que ha tenido en Pe como consecuencia, nos hace pensar en dos cuestiones. La primera es acerca de su nivel de respuesta frente a las situaciones. Hemos podido comprobar que al recibir la pulsera se ha mostrado sumamente agradecido y feliz; por ello, tal vez se muestre dispuesto a corresponder ante determinadas propuestas.

De esto se desprende la segunda cuestión: parece estar dispuesto al vínculo, (lo que se manifiesta hasta ahora por el que mantiene con su hermana C). Esta necesidad de apego ha de ser un elemento muy importante para tener en cuenta para adaptar el modo particular de inferir un proceso de enfoque arteterapéutico con él. Durante la interacción simbólica que se produzca con el usuario, prestaremos atención a las posibilidades de significación particular que atribuye nuestro protagonista a cada hecho. La observación 
de primera mano hacia lo cotidiano, la proximidad y una estructura no impuesta de forma previa dará lugar a una dinámica abierta que se irá construyendo conforme ocurre, facilitando un material de estudio que se generará en vivo, manteniendo un diálogo permanente e intersubjetivo.

Hablamos de un vínculo que puede llegar a emerger con la figura del acompañante en el taller, si al ceder "una pulsera" se produce posteriormente un momento de juego entre ambos. ¿Por qué el juego como instrumento? Paz Martínez (2017), en la línea de Winnicott (1971) lo sitúa "entre la realidad psíquica e interna y la externa, en el espacio transicional, donde se desarrollarán el juego y la capacidad creadora como fenómenos transicionales" (p.193). Justo en esta zona, en el espacio transicional entre ambas realidades, es donde actúa el arteterapia, por lo que debe de ser algo a tener en cuenta para la relación con el usuario elegido; sabemos dónde actuar y un modo de hacerlo es el juego.

Se elige la técnica de garabato de Winnicot, squiggle ${ }^{3}$. Muchos autores, entre ellos Günter (2008) y Martínez (2017), aseguran que se trata de una terapia con la paciente mucho más divertida, aunque detrás tenga la profesionalidad y reflexiones propias del terapeuta, que permitirá ofrecer un carácter lúdico a la sesión y hará al paciente olvidarse del resto de sus problemas, vaciarse en el papel, a la vez que se sincera y desahoga. El terapeuta, o en nuestro caso la guía del taller tendrá la misma intención que la del paciente, la de jugar también, pues si él o ella no lo hace, el paciente tampoco.

Aun así se debe ser consciente en todo momento de las relaciones transferenciales que se producirán entre ambos, entendidas por muchos autores como Jung (1993) como fenómenos que aparecen en la relación paciente-psicoanalista que implican, en ocasiones, problemas en la terapia. Freud (1917), explicaba casos en los que un paciente transfería sobre la persona del médico sus representaciones inconscientes. Distingue dos transferencias: una positiva y otra negativa, con sentimientos de ternura y sentimientos hostiles. En arteterapia, el registro en un diario de las sesiones es la clave para observar las posibles transferencias y contratransferencias que puedan pasarse por alto, por lo que las anotaciones en esta experiencia son imprescindibles.

Si lo que se plantea se lleva a cabo de forma exitosa, se conseguirá que un objeto (la pulsera) simbolice el vínculo que mantienen las dos personas implicadas en este proceso. Para ello solicitamos permiso al centro, pues la estrategia puede estar en contradicción con las intervenciones acerca de las "posesiones" de Pe, (recordemos que se las retiran con cierta frecuencia), asegurando el seguimiento y supervisión del proceso como parte de un trabajo de investigación.

A pesar de que el taller fue más extenso, serán diez las sesiones dedicadas al acompañamiento a Pe en la creación, en la que se darán las siguientes fases:

\footnotetext{
${ }^{3}$ Squiggle: El objetivo terapéutico de esta técnica de Winnicott, era "desplegar la capacidad de jugar del paciente, su gesto espontáneo y desde un encuadre humano, crear con él una comunicación profunda en la que se sienta comprendido y alentado de que el terapeuta le puede ayudar, así como favorecer su integración psíquica" (Lacruz, 2010, como se citó en Martínez, 2017, p.193). Dando más importancia al niño que juega, el proceso y la capacidad de jugar, que a el juego y su trama (Martínez, 2017).
} 


\section{Una pulsera como desencadenante del juego}

Pe comienza la sesión muy tranquila, pide su formato pequeño habitual para comenzar a dibujar sus "casas de C". Esta serenidad termina cuando parece recordar que el día anterior le regalaron una pulsera y empieza a pedir otra de forma insistente. No hay ninguna pulsera en el taller y le informamos de ello, pero aún así se empeña en que localicemos una. Con su impaciencia, la calma inicial de la sesión ha desaparecido completamente, incluso para los/as compañeros/as que se contagian fácilmente de su estado de ánimo. Tomamos un simple cordel que atamos a su muñeca, y la emoción lo inunda de nuevo como la primera vez que esto sucedió. Busca contacto visual y verbal con el que transmitir su agradecimiento, y en este momento encontramos la excusa perfecta para comenzar a jugar. La guía del taller le propone: “¿Quieres que juguemos ahora que tienes tu pulsera?" A lo que responde ilusionado: "iSi! Vamos a hacer un juego".

Como ya hemos dicho anteriormente, la intención es establecer un momento de juego a través de la técnica de Winnicott, squiggle. El juego en su origen es para dos personas. Consiste en que uno de los miembros de la pareja hace un garabato con los ojos cerrados y, a continuación, el otro lo cierra creando una forma, reconocible o no, y así sucesivamente, alternando el orden de intervención. Si se logra que Pe se interese por esta propuesta, en función de su carácter lúdico, es posible que surjan nuevas posibilidades de interacción y en consecuencia, quizás con ello se produzca una ampliación de su alfabeto gráfico y sus redes significativas.

Durante las siguientes sesiones, no solo se intentará mantener dichos encuentros, sino también que este venga simbolizado por la cesión de una pulsera. Esta continuará en su poder cuando la guía del taller no esté presente, desplazando metonímicamente su presencia al objeto. Se buscará de este modo establecer un vínculo entre ambos participantes del juego, en el que el usuario pueda experimentar una "presencia en la ausencia".

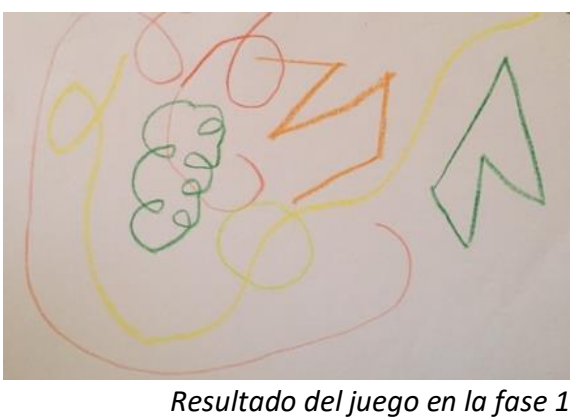

A través de pautas sencillas para que la estrategia se adapte a sus capacidades, comienza el juego. Procede tal como indica Winnicott en sus entrevistas, la pareja terapeuta-paciente, (en nuestro caso, guía-usuario) se sentará a jugar, uno bosquejará una forma con los ojos cerrados y el otro lo completará creando algo reconocible, y así se produce una "creación transicional de autoría compartida" (Lacruz, 2011).

Sobre folios en blanco, y una frente a otro, Pe es retado con un garabato, que decide cerrar. ¿Por qué elige esa forma que define un espacio cerrado y no otra? Se decide preguntarle a él mismo, a lo que responde: "Porque es una pulsera", y a la vez sonríe. Con este gesto, y siendo ajeno a los objetivos de esta investigación, confirma que él también parece otorgar un valor simbólico a dicho objeto. Líneas y curvas ocupan el formato una y otra vez, que interrumpe con un: "Gracias por darme la pulsera", poniendo énfasis en el por qué de lo que estamos haciendo.

Al introducir formas reconocibles, tal y como esperábamos ha dibujado una casa, pero no la define como "la casa de mi C", parece un simple gesto para impresionar, o bien sentirse seguro, ya que es el elemento que mejor sabe hacer. Observamos que la siguiente forma y las que le siguen, vuelven a ser la misma. En cualquier caso, el juego ha sido un momento divertido para él y parece haberlo disfrutado. 


\section{Interferencias en el proceso}

La última pulsera fue un cordel, por lo que corremos el riesgo de que la pierda. Riesgo en el sentido de que como hemos dicho, Pe se vincula a los objetos que valora, y cuando esto sucede afecta en gran medida a su estado de ánimo. Por lo tanto, el cordel se convertirá en una pulsera real, que estará presente en cada sesión. Lo primero que ha hecho el usuario al entrar al taller ha sido pedirla.

En esta ocasión, el ambiente entre sus compañeros/as es algo tenso, están intranquilos/as. La monitora nos informa que puede ser porque uno de ellos ha enfermado y salido de la Casa Familiar. Aún así, esto no parece afectar mucho a Pe, que parece muy activo y expectante. Al preguntarle: “¿Recuerdas qué hicimos cuando te dí la pulsera?" Responde: "Sí, ¿jugamos?" El valor simbólico de dicho objeto comienza a ser evidente.

Parece que la atmósfera inestable interfiere en la actividad, en la que únicamente dibuja una de sus formas habituales, sin dejarse llevar por la espontaneidad del garabato. A pesar de su distracción, entendemos que estos vaivenes también forman parte del proceso, ya que aunque no centre su atención en el juego, está sentado con la intención de hacerlo. Incluso se mira la pulsera, le gusta mucho y lo hace saber. Esta situación nos hace conscientes de la fuerza que empieza a tener el vínculo intersubjetivo: por un lado, porque a pesar de que las circunstancias no son las óptimas, Pe se muestra dispuesto a complacer a la guía, en tanto que esta siente por primera vez de forma palpable el valor de la contratrasferencia, que se muestra en cierto grado de frustración ante lo que considera en ese momento una sesión poco exitosa. Su propia emoción no le pasa desapercibida; lejos de ser un obstáculo se convierte en un instrumento de medida de la relación transferencial.

\section{Producciones que vinculan y reconocen la realidad}

Su entrada al taller ha sido fascinante, con un exclamativo " $A$ p pintar se ha dicho!" Se sienta junto a quien considera ya su "confidente", le muestra las pulseras que tiene puestas y guiñando dice "Es nuestro secreto".

Traemos preparada la siguiente pulsera. Cuando la ve, le encanta y se la ofrecemos, a lo que inesperadamente responde: "No, esa es para ti, te la pones tú y yo me pongo esta" Las transferencias son claras en este momento del proceso, pues el usuario ha sentido la necesidad de reforzar el vínculo con este gesto. Cada uno tiene dicho objeto simbólico; las dos pulseras se han convertido en signo de lo que sucede. Su conversación no cesa y al mirar ambas dice: "Que bonitas, es que somos amigos y jugamos." Se ríe de forma divertida y se retoma el juego.

Él mismo ha tomado la iniciativa en la creación, traza un garabato y lanza una mirada divertida a la vez que desafiante, "iTe toca!". Su atención e interés está ayudando a la fluidez de la sesión. Se dejan de lado los trazos aleatorios, y se producen formas reconocibles, en los que él también participa copiándolos. De este modo se comienza a producir una obra conjunta en la que ambos fusionan sus trazos, y a la que él titula: "Las pulseras". En cierto modo, es como si estuviera realizando un retrato del vínculo.

Este tipo de producciones, protagonizan el resto de la sesión y permiten a sus participantes evadirse del resto del grupo. Esto motiva a $\mathrm{Pe}$ a

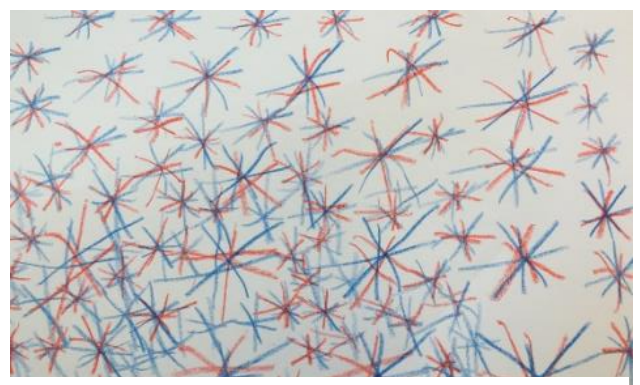

"Las pulseras" titulado por Pe en la fase 3 
sincerarse con su acompañante: "Yo creo que mi C me quiere mucho, pienso que sí, aunque no me lo diga mucho, pero creo que será así". Se adentran en una conversación, que Pe no suele tener con nadie, como después nos aseguran en el centro. Quizá no haya sido una gran declaración, pero lo que más importa en este momento es lo que lo ha llevado a hablar de este modo, en un espacio de seguridad y confidencialidad donde sus pensamientos están a salvo. Aquí vemos la importancia del proceso en arteterapia, en el que suceden cosas como esta, que proceden directamente de la vida psíquica del usuario. De este modo podemos constatar cómo la actividad artística en el taller le está permitiendo evolucionar en su capacidad de vínculo y de autorreconocimiento acerca de su propia realidad.

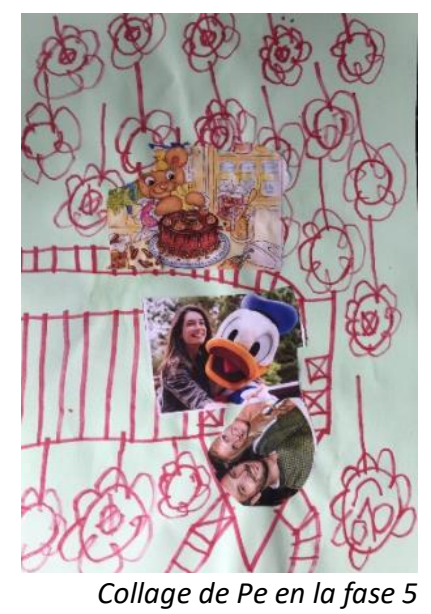

\section{Proyección de emociones}

En la siguiente fase del proceso, la guía del taller decide no proponer el intercambio de la pulsera ni del juego, para observar lo que sucede. Efectivamente, Pe la busca para pedirle cambiar las pulseras. Acto seguido dice entusiasmado: "Ya si, vamos a jugar". Esta vez a través de formas reconocibles, la acompañante comienza dibujando elementos que significan algo en su vida, a la vez que se lo cuenta a Pe. Este responde con el dibujo de un coche, que son su hermana $\mathrm{C}$ y su otro hermano, que se van. Tal vez asocia esto a los momentos de despedida cuando sus seres queridos lo visitan, pero después se van. También representa una moto, en la que según cuenta se montaba con su hermano.

Está narrando episodios del pasado, esa necesidad que todos/as los/as usuarios/as tienen de mantener presente su vida anterior, siente la posibilidad de hacerlo con quien ahora considera su "confidente". Como dice Martínez (2017), durante este proceso "el paciente se sentirá comprendido y sabrá que el terapeuta lo puede ayudar". Comenzamos a experimentar un tipo de conexión que, según Winnicott, se origina en la relación del niño con su madre (Günter, 2008). Al igual que el/la niño/a proyecta sus emociones en la madre, el/la paciente lo podrá hacer con el terapeuta. En nuestro caso, como sabemos, no hay terapeuta y paciente sino guía y usuario, pero de forma paralela, parece que $\mathrm{Pe}$ comienza a sentir que el espacio vincular es un lugar de sostén, suficientemente confiable para funcionar como tal.

\section{Sentirse capaz gracias al "Equipo de las pulseras"}

La motivación por crear de forma conjunta en cada una de las sesiones va en aumento. Pe no había querido probar anteriormente ninguna técnica que no fuese el dibujo, pero al ver que uno de los compañeros está trabajando el collage, decide probar. Hoy no ha buscado establecer un momento de juego, pero no para de llamar a su acompañante buscando su aprobación. Ha dibujado sus casas, pero ha pegado en el interior fotografías de personas. Dice: "iMira, mira! Este soy yo con mi hermana $C$ en nuestra casa, y estás tú en la casa también".

La relación vincular ha llegado a un momento clave en el proceso, pues es lo bastante fuerte para que el usuario conciba a la guía en ese espacio entre su mundo exterior e interior; hace sitio para alguien más. Reconoce a la guía del taller dentro de su espacio emocional privado. Los resultados 
artísticos de este momento del proceso, son verdaderamente interesantes, pues su producción pasa a ser tan nueva e inesperada en él, que hasta el mismo Pe se sorprende y siente orgulloso de lo que ha hecho: "Qué bonito lo que hemos hecho" repite al terminar cada dibujo. Podría ser que no solo se refiera al dibujo, sino al bienestar que siente en el taller y en su proceso.

Nuestra sensación parece corroborarse cuando, al sentarse junto a la guía dice: "Te quiero mucho, somos el equipo de las pulseras". Hoy el juego no se ha producido con garabatos y formas, pero sí comentando su experiencia con esta nueva técnica. Tampoco lo ha estado la pulsera de forma física, pero ha adquirido un valor fundamental con la última expresión de Pe.

En la siguiente sesión se ha ofrecido él mismo a

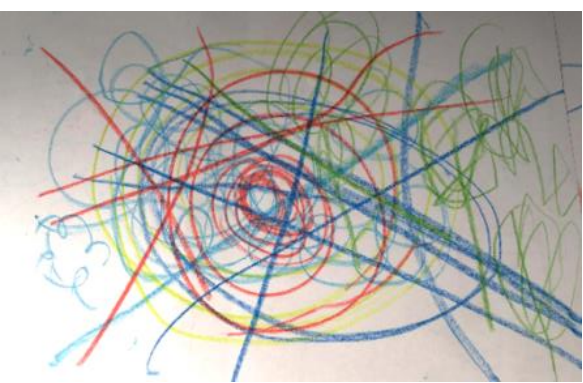

Pintura de Pe en la fase 5 probar una nueva técnica: la pintura. Su implicación en el taller ha aumentado con el paso de los días, y se siente capaz de tener iniciativas y complacer a esa persona con la que ha creado un vínculo.

\section{Reforzar el vínculo ya creado}

Este momento también se convierte en clave del proceso, de modo sobresaliente, ya que el usuario pide que se intercambien las pulseras, pero no con la de siempre. Desde que lo conocimos, tiene siempre una pulsera que no se quita nunca, y esa es la que quiere intercambiar como si quisiese reforzar el vínculo creado. La monitora escucha esta conversación y se sorprende muchísimo de que la ofrezca, ya que cuenta que jamás han conseguido que se desprenda de ella. Ofrece por última vez jugar al "juego de las pulseras" en el que, con risas, garabatos con fuerza y sin más preocupación, se divierten creando. Winnicott habla de la "afección a estos momentos como si de algo

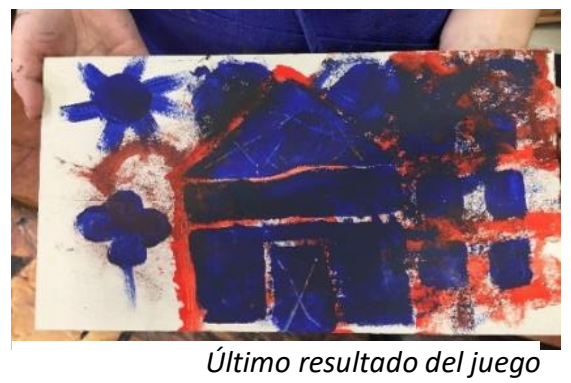
sagrado se tratase". Nos divertimos y disfrutamos, como vemos que sucede con el usuario, el cual identifica este momento del juego como algo sagrado, simbolizado por una pulsera y que hay que hacer antes de dar por finalizada la sesión.

\section{Conclusiones}

Como resultado a la técnica del garabato o squiggle, aplicada a lo largo de las sesiones, y una metodología basada en el acompañamiento, se han podido confirmar los efectos que puede llegar a tener el arteterapia y cumplir los objetivos que guiaban la investigación.

Referido al primer objetivo que nos planteábamos, inferir un proceso de arteterapia a partir del juego del garabato de Winnicott, podemos afirmar que todo el proceso ha sido guiado por dicha técnica además de las bases y fundamentos teóricos del arteterapia. Gracias a esto se ha conseguido acompañar a Pe en un proceso arteterapéutico a través del juego, que acogió esta dinámica de autoría compartida y carácter lúdico con mucho entusiasmo, llegando a convertirse prácticamente en un momento sagrado para él. EI acompañamiento que se buscaba en el segundo objetivo ha traído consigo no solo la confianza necesaria para un reconocimiento de su propia realidad, sino también la creación de un nuevo alfabeto de formas en su producción, que le abren posibilidades de reflexión hacia su propia vida. Además, hemos podido constatar que a partir de la 
experiencia se produce en él un empoderamiento que se traduce en capacidad y motivación para experimentar con nuevas técnicas artísticas como el collage o la pintura. La actividad no pretende inducir al aprendizaje de técnicas artísticas, aunque este pueda ser una consecuencia de ello, pero la posibilidad de practicarlas se ha convertido en el detonante para que Pe tome la iniciativa de su propio proceso, así como en soportes para su expresión, que de ese modo se diversifica, evoluciona y se expande hacia nuevas ideas.

En cuanto a la necesidad de establecer vínculos transferenciales entre usuario y guía del taller de arteterapia mediante el reconocimiento de fenómenos transicionales, que observábamos como tercer objetivo, podemos confirmar que las transferencias activas y pasivas con el paciente han estado presentes en las sesiones, apareciendo de forma progresiva, aumentando conforme se reforzaba el vínculo entre ambos. Las emociones que evocaba el momento lúdico han sido detectadas gracias al reconocimiento de estos fenómenos transicionales. Esto ha permitido el refuerzo del vínculo, adaptando el proceso completamente a sus necesidades. Durante la experiencia podemos ver como ha sido él mismo el que ha decidido las pautas de la investigación, la guía solo ha debido captarlas y la estructura no ha sido impuesta, sino que se ha ido descubriendo sola, desde la espontaneidad de lo acontecido.

El cuarto objetivo señalaba la posibilidad de utilizar el juego como estrategia para reforzar los vínculos hacia la actividad y las personas implicadas en el proceso. La experiencia ha demostrado que ha sido este momento lúdico el que ha permitido que se forme un vínculo simbólico. Pe ha depositado su confianza en la guía del taller, considerándola como su "confidente" con la que puede abrirse de forma segura y libre, ya sea a través del trazo o de la palabra. Hay que destacar que esta zona donde dialogar de subjetividad a subjetividad, ha sido posible siempre y cuando los factores externos no hayan interrumpido la canalización de las emociones. Por lo tanto, llegamos a la conclusión de que esto ha funcionado, en la medida en que se ha podido mantener el encuadre terapéutico.

Además de los objetivos planteados inicialmente, el proceso nos lleva a lograr cuestiones con las que no contábamos. Efectivamente para que el juego haya sido bien recibido por el usuario, se ha debido encontrar el modo de llamar su interés, facilitado a través de factores inesperados de la espontaneidad de Pe. Aparece un objeto, "la pulsera", que acaba funcionando como un objeto transicional que lo mantiene unido a la actividad que realiza con la figura de guía en el taller. Ha acabado representando el vínculo entre ambos como consecuencia del momento lúdico. El significado que tenían para él anteriormente estos objetos ha cambiado; han pasado de llamar simplemente su atención a significar algo tan fuerte como es una relación vincular. En efecto, simbolizan la relación que se ha establecido; aún siendo algo tan aparentemente simple, ha ocupado un lugar de crucial importancia en el proceso. La pulsera pasa a ser un elemento que le hace tener consigo la presencia de su acompañante cuando no se está produciendo el juego: una presencia en la ausencia, concepto del que partió la investigación.

Por esta razón, nos atrevemos a decir que la metodología usada ha sido la adecuada: a partir de la observación y anotación de los acontecimientos producidos en el trabajo de campo; el análisis de la conducta del usuario y su significado, y la indagación y constatación en las lecturas que hablan sobre los conceptos que aparecían durante la experiencia. Una vez asimilados, se posibilita la comprensión de su proceso, lo cual ha beneficiado a la creación del vínculo.

Otra cuestión que es necesario enfatizar es que en ningún caso se ha pretendido hacer arteterapia, sino plantear una actividad siendo conscientes de que el proceso artístico dentro de una relación vincular acompañante, puede tener efectos terapéuticos. 
Por lo tanto, consideramos que se han cumplido los objetivos, además de ir aún más allá en el vínculo que se preveía conseguir con el transcurso de las sesiones. Ha sido sorprendente ver la evolución del usuario y cómo han sido logrados y/o superados los propósitos de los que se partía.

Para concluir, nos atrevemos a decir que la hipótesis inicial no sólo se ha cumplido, sino que nos ha procurado mucho más. En efecto, podemos confirmar que "el arteterapia es un vehículo posible para el establecimiento de vínculo simbólico, en especial con una dinámica de juego según la técnica del garabato de Winnicott, en un contexto de discapacidad intelectual y problemas de conducta", pero además hemos podido aprender y disfrutar de las cualidades de esta actividad y este vínculo enriquecedor, que nos han proporcionado recursos, conocimiento, experiencia, capacidad de análisis y reflexión, en el campo del arte en tanto expresión

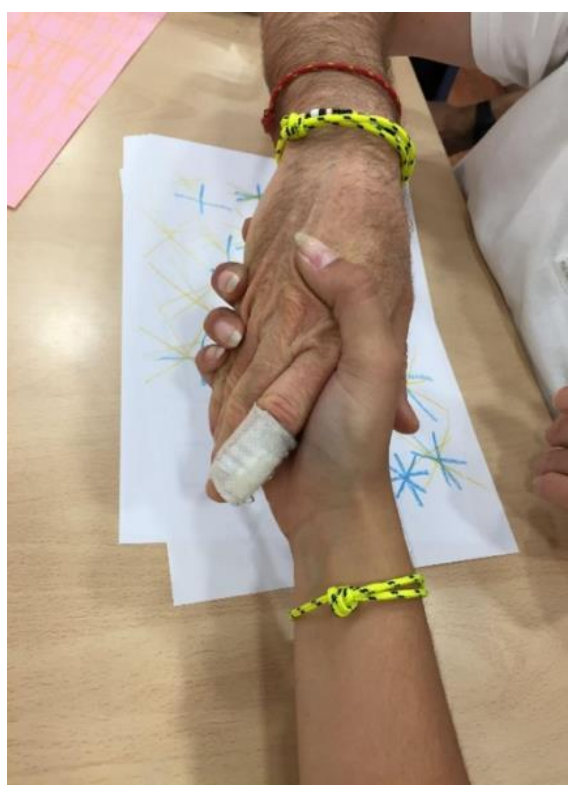

Pe y guía del taller en la última sesión humana como en su dimensión terapéutica, confirmándose, como afirma Kavafis (1911), que la belleza del viaje y lo hallado mientras sucede, supera cualquier Ítaca.

Referencias

Abadi, S (2014). Transiciones, el modelo terapéutico de D.W. Winnicott. Cauces Editores.

Ávila Espada, A (2015). Del encuadre como factor técnico a la intersubjetividad del vínculo terapéutico. Clínica e Investigación Relacional, 9(2), 394-397.

Avilés Martos, M y López Pérez, E (2014). Jugar con trampa. Taller de Arteterapia en el Centro de Día de Atención a Menores de Aldeas Infantiles SOS Granada. Papeles de arteterapia y educación artística para la inclusión social, 9, 73-86.

Bassols, M (2006). El arteterapia, un acompañamiento en la creación y la transformación. Papeles de arteterapia y educación artística para la inclusión social, 1, 19-25.

Federación Española de Asociaciones Profesionales de Arteterapia. (29 de mayo de 2021). Formaciones.

Freud, S (1917). Introducción al psicoanálisis. Italia: Newton Compton Editori.

Günter, M (2008). Colloqui con i bambini, la tecnica dello scarabocchio nella pratica clinica. Ubaldini Editore.

Jung, C.G (1993). La psicología de la transferencia: esclarecida por medio de una serie de imágenes de la alquimia. Ediciones Paidós.
Lacruz, J (2011). Donald Winnicott: vocabulario esencial. Mira Editores.

Laplanche, J y Pontalis, J (1996). Diccionario de psicoanálisis. Ediciones Paidós.

López, M y Martínez, N (2006). Arteterapia. Ediciones Tutor.

Lowenfeld, V y Brittain, W.L (1980). El desarrollo de la capacidad creadora. Kapelusz.

Machado, A (1912). Proverbios y Cantares: Campos de Castilla. El País.

Martínez Loné, P (2017). El garabato de Winnicott y su uso inspirador en arteterapia. Papeles de Arteterapia y educación artística para la inclusión social, 12, 191-203.

Masini Fernández, C (2015). Psicodrama. Reflexiones desde la práctica clínica en un Hospital de Día Psiquiátrico. Papeles de Arteterapia y educación artística para la inclusión social, 153-164.

Ortega Cubero, I (2014). Repetición, estereotipo y dibujo infantil. Papeles de arteterapia y educación artística para la inclusión social, $9,125-144$.

Winnicott, D (1971). Realidad y Juego. Editorial Gedisa SA 